\title{
Enhanced physical aging of polymer nanocomposites: the key role of the area to volume ratio
}

\author{
Daniele Cangialosi, ${ }^{1}$ Virginie M. Boucher, ${ }^{2}$ Angel Alegría, ${ }^{1,3}$ Juan \\ Colmenero $^{1,2,3}$ \\ ${ }^{1}$ Centro de Física de Materiales (CSIC-UPV/EHU), Paseo Manuel de Lardizabal 5, \\ 20018 San Sebastián, Spain, ${ }^{2}$ Donostia International Physics Center, Paseo Manuel de \\ Lardizabal 4, 20018 San Sebastián, Spain, ${ }^{3}$ Departamento de Física de Materiales, \\ Universidad del País Vasco (UPV/EHU), Apartado 1072, 20080 San Sebastián, Spain
}

\begin{abstract}
The physical aging process, namely the spontaneous evolution of the thermodynamic state occurring in glasses, has been monitored in poly(methyl methacrylate) (PMMA)/silica and polystyrene (PS)/silica nanocomposites following the time evolution of the enthalpy by means of differential scanning calorimetry (DSC). The effect on physical aging of the content of silica particles has been investigated in details varying it over a wide range. Our results indicate accelerated physical aging in the nanocomposites in comparison to the corresponding pure polymer. Furthermore the acceleration is generally more pronounced in nanocomposites presenting a high silica content. This result cannot be attributed to a difference in molecular mobility of the polymers in the nanocomposites in comparison to pure PMMA and PS, since broadband dielectric spectroscopy (BDS) indicates no effect of silica nanoparticles on the polymer segmental dynamics. Moreover, calorimetric measurements reveal a reduction of the heat capacity jump at $T_{g}$ for the nanocomposites, as well as lower experimentally recoverable enthalpy values. This may result from the faster non-isothermal evolution of the glass state when cooling down the samples from their liquid state. To account for these experimental results the acceleration of physical aging has been rationalized in the framework of the diffusion of free volume holes model as previously proposed. This is able to adequately catch the dependence of the physical aging rate on silica content, since this determines the area of nanoparticles to the volume of polymer that is the relevant variable within the diffusion model.
\end{abstract}


Keywords: Enthalpy relaxation, Glass transition temperature depression, Segmental mobility, Diffusion model, Nanocomposites

\section{Introduction}

Polymer nanocomposites have increasingly captured the attention of the scientific community in the last decade. They owe their success to the specific surface area, which may favorably modify the properties, thus rendering polymer nanocomposites amenable to be employed in a wide range of applications and attractive from a fundamental point of view $[1,2]$.

In recent years, increasing attention has been devoted to the study of the slow spontaneous evolution of the thermodynamic state occurring in the glassy matrix of polymer nanocomposites, with particular emphasis to the differences of such evolution in comparison to the corresponding "bulk" polymer [3]. This process, known as physical aging, has been intensely studied for pure glassy polymers $[4,5]$. It is generally believed that physical aging in bulk glassy polymers is associated to the molecular mobility in the glass [6].

The latter idea has been recently questioned when monitoring the physical aging of poly(methyl methacrylate) (PMMA)/silica [7, 8, 9] and polystyrene (PS)/gold nanocomposites [10]. In these systems, a decoupling between the rate of physical aging and the segmental dynamics has been found. In particular, physical aging in the nanocomposites has been observed to be accelerated in comparison to the corresponding bulk polymer, whereas no effect of the presence of silica nanoparticles has been found on the polymer segmental dynamics. Thus the acceleration of physical aging in polymer nanocomposites suggests that, further than molecular mobility, additional factors control the rate of physical aging. In the case of polymer nanocomposites, the filler content that determines the amount of specific surface area, i.e. the area of nanoparticles to the volume of polymer ratio, may be of importance, as suggested by our previous publications $[7,8,9,10]$. Nonetheless the range of variation of filler content in those studies was too limited to draw definitive conclusions. Moreover the free volume holes diffusion model was able to rationalize the increase of the rate of physical aging found in PMMA/silica nanocomposites [7, 8, 9] and PS/gold nanocomposites [10]. A similar approach was employed to describe physical aging in polymer thin films with a thickness in the micrometer range $[11,12,13,14,15,16,17]$. The latter systems also display a notable acceleration of the rate of physical aging with decreasing the film thickness. 
In this work, we focus our investigation on the effect of the area to volume ratio of PMMA/silica and PS/silica nanocomposites on the rate of physical aging. To do so, we have systematically varied the silica content in the polymer in a wide range and monitored the physical aging process following the time evolution of the enthalpy as measured by differential scanning calorimetry (DSC). Our results show that the trend of acceleration of physical aging previously observed $[7,8,9,10]$ is maintained over a wide range of filler contents. Furthermore such acceleration is accompanied by a depression in calorimetric glass transition temperature $\left(T_{g}\right)$ and a reduction in the specific heat jump at $T_{g}$. The main consequence of the acceleration of physical aging is that polymer nanocomposites with higher silica content display only a part of the recovered enthalpy, due to the delay time intrinsic to calorimetric measurements. In the case of significantly accelerated physical aging, this delay does not allow monitoring the first stages of enthalpy recovery. This issue is particularly important during the cooling process in proximity of $T_{g}$, where the non-isothermal aging can be significant due to the relatively high mobility in the glass. As we do not find any effect of the presence of silica nanoparticles on PMMA and PS segmental dynamics (except for the most concentrated PS nanocomposite), as measured by broadband dielectric spectroscopy (BDS), we interpret the acceleration of physical aging in the framework of the diffusion of free volume holes model, recently revitalized to explain such acceleration in nanostructured systems $[18,19,16,17,20,21,7,8,22]$.

\section{Experimental}

PMMA and PS were purchased from Polymer Source, Inc. The molecular weights were respectively $\mathrm{M}_{w}=120000 \mathrm{~g} \mathrm{~mol}^{-1}$ and $M_{w}=85000 \mathrm{gmol}^{-1}$ and the polydispersity $\mathrm{M}_{w} / \mathrm{M}_{n}=1.1$ and $\mathrm{M}_{w} / \mathrm{M}_{n}=1.02$. Nanoparticles were Nan-O-Sil amorphous colloidal silica provided by Energy Strategy Associates, Inc. These are spherical particles with an average diameter of $100 \mathrm{~nm}$. Prior to mixing with the polymers, silica nanoparticles were functionalized in tetrahydrofuran (THF) with Octadecyltrimethoxysilane (OTMS) to avoid particles aggregation. The polymers and functionalized silica nanoparticles were subsequently solution blended in THF. The solution was then cast onto a clean glass substrate warmed up for the solvent to evaporate more rapidly and left in a fume hood for at least $15 \mathrm{~h}$. The films were finally put in a vacuum oven at $T_{g}+30{ }^{\circ} \mathrm{C}$ for at least $72 \mathrm{~h}$ in order to eliminate all traces 


\begin{tabular}{|c|c|c|c|c|c|c|c|}
\hline Sample & $T_{g}\left({ }^{\circ} \mathrm{C}\right)$ & Silica wt. \% & Silica vol. \% & $\epsilon^{\prime \prime} \max (\alpha)(10 \mathrm{~Hz})$ & $\epsilon^{\prime \prime} \max (\beta)(10 \mathrm{~Hz})$ & $\epsilon^{\prime \prime} \max (\mathrm{T})^{*}$ & \% Eq. thick. $(\mathrm{nm})^{* *}$ \\
\hline PMMA & $118 \pm 0.5$ & - & - & 0.097 & 0.135 & 0.26 & 2000 \\
\hline M10 & $117.8 \pm 0.5$ & 10 & 5 & 0.096 & 0.13 & 0.265 & 1180 \\
\hline M17 & $116.6 \pm 0.5$ & 17 & 8 & 0.098 & 0.13 & 0.26 & 700 \\
\hline M24 & $117.3 \pm 0.5$ & 24 & 12 & 0.097 & 0.1 & 0.265 & 380 \\
\hline M38 & $115.8 \pm 0.5$ & 38 & 19 & 0.096 & 0.12 & 0.27 & 230 \\
\hline M52 & $115 \pm 0.5$ & 52 & 26 & 0.097 & 0.095 & 0.26 & 170 \\
\hline$\overline{\overline{P S}}$ & $\overline{103.2 \pm 0.5}$ & - & - & 0.0068 & - & $\overline{0.0067}$ & 8000 \\
\hline S1 & $102.4 \pm 0.5$ & 1.1 & 0.4 & 0.0068 & - & 0.007 & 3900 \\
\hline S3 & $101.9 \pm 0.5$ & 2.8 & 1.1 & 0.0067 & - & 0.007 & 2160 \\
\hline S9 & $100.6 \pm 0.5$ & 9.5 & 4 & 0.0072 & - & 0.007 & 1200 \\
\hline $\mathrm{S} 24$ & $103.4 \pm 0.5$ & 24.5 & 11.4 & 0.007 & - & 0.007 & 450 \\
\hline
\end{tabular}

*The temperature is 150 and $130{ }^{\circ} \mathrm{C}$ for PMMA and PS respectively. ${ }^{* *}$ Obtained from the fitting of the diffusion model.

Table 1: Calorimetric $T_{g}$ on cooling at $20{ }^{\circ} \mathrm{C} \mathrm{min}^{-1}$, silica weight and volume percentages, normalization factors for the $\epsilon$ " displayed in Figs. 3 and 4 and equivalent thicknesses for all investigated samples.

of solvent. The silica content in the nanocomposite samples was measured by thermogravimetric analyses (TGA), on a thermogravimeter TA Q-500, with a heating ramp of $10{ }^{\circ} \mathrm{C} \min ^{-1}$ up to $750{ }^{\circ} \mathrm{C}$ under flowing nitrogen $\left(20 \mathrm{~cm}^{3} \mathrm{~min}^{-1}\right)$. The values of silica weight fractions and the corresponding volume fractions are reported in Table 1.

TEM measurements were performed using a high-resolution transmission electron microscope TECNAI G2 20 TWIN. The measurements were carried out using an accelerating voltage of $200 \mathrm{kV}$, under low dose conditions. Nanocomposites ultra-thin sections of about $70 \mathrm{~nm}$ were cut by a LEICA EM UC6 microtome equipped with a diamond knife and placed on a 400-mesh copper grid. Fig. 1 shows representative transmission electron micrographs for PMMA/silica nanocomposites M10 and M17 and for PS/silica nanocomposites S10 and S24. A general increase of nanoparticles aggregation with increasing silica content can be observed, though this seems to be more marked in PMMA nanocomposites.

The molecular dynamics of all nanocomposites and the pure polymers was obtained by means of broadband dielectric spectroscopy (BDS) that allows measuring the complex dielectric permittivity: $\epsilon^{*}=\epsilon^{\prime}(\omega)-i \epsilon^{\prime \prime}(\omega)$, where $\epsilon^{\prime}$ and $\epsilon^{\prime \prime}$ are respectively the storage and loss part of the dielectric permittivity, in a frequency range $10^{-2}-10^{7} \mathrm{~Hz}$. Samples with thickness around $200 \mu \mathrm{m}$ were placed between two gold-plated electrodes and introduced in the dielectric cell. The temperature was controlled by a Novocontrol Quatro cryosys- 

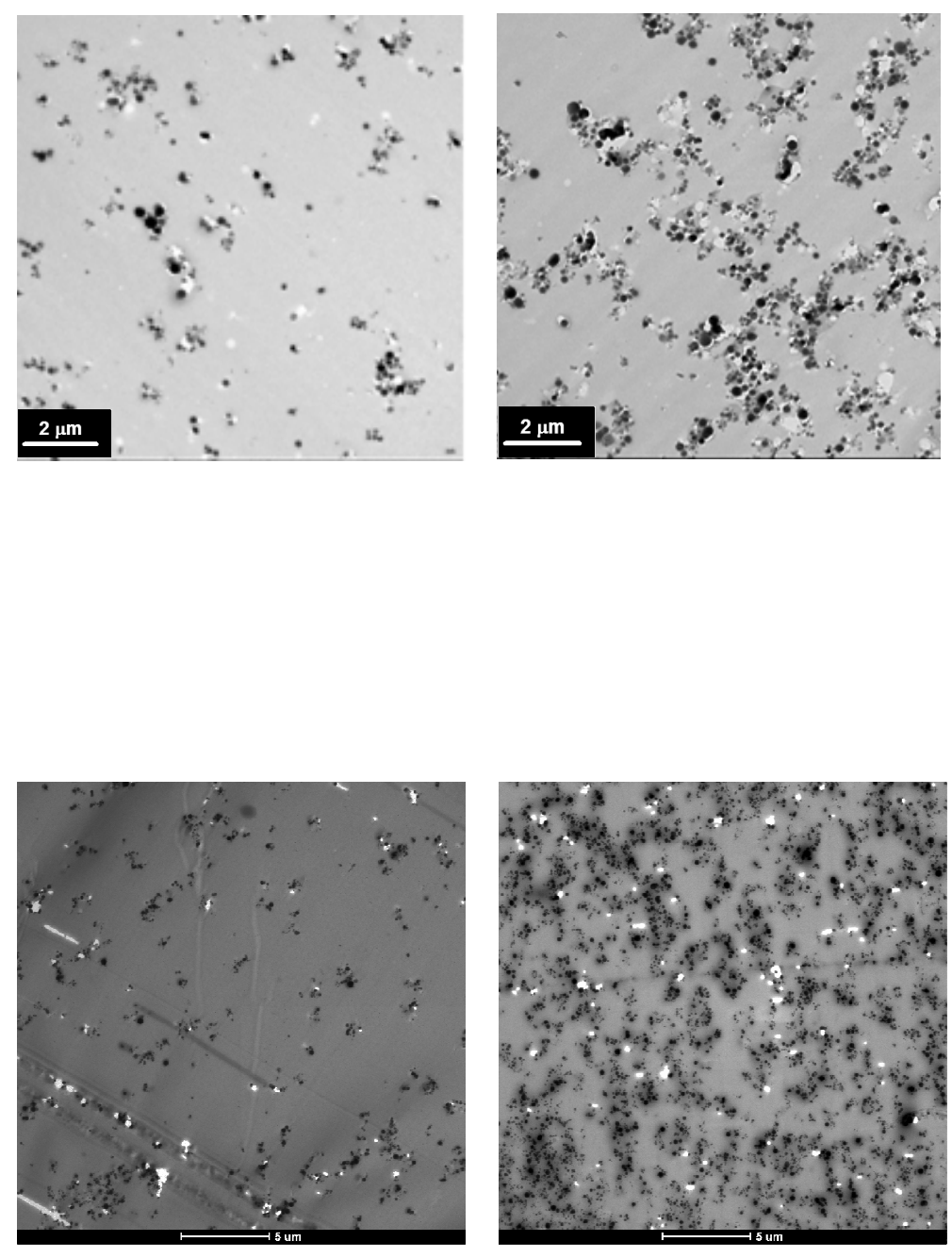

Figure 1: TEM images of the samples labelled as M10 and M17 (upper panel); and S10 and S24 (lower panel). 


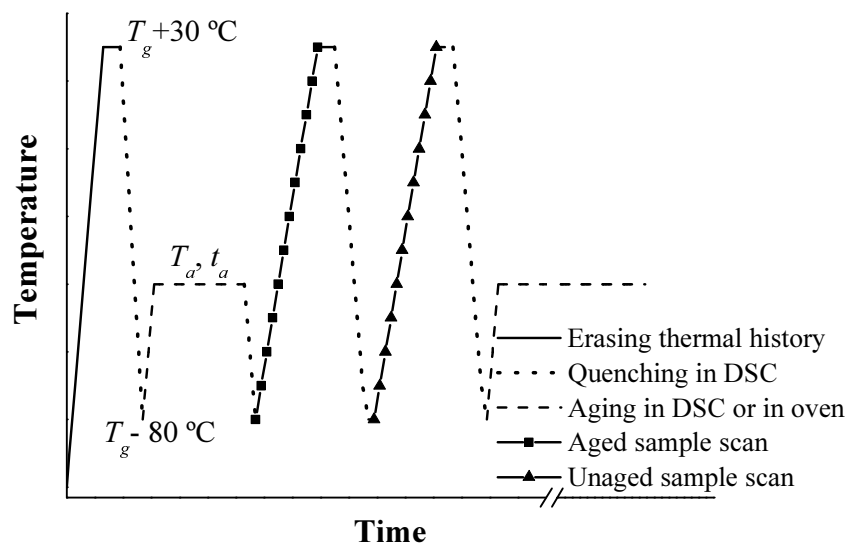

Figure 2: Thermal history for enthalpy recovery experiments.

tem with a precision of $\pm 0.1 \mathrm{~K}$. Frequency scans were recorded on all samples isothermally in steps of $5{ }^{\circ} \mathrm{C}$ from 155 to $0{ }^{\circ} \mathrm{C}$ for PMMA/silica nanocomposites and in steps of $2.5{ }^{\circ} \mathrm{C}$ from 150 to $70{ }^{\circ} \mathrm{C}$ for PS/silica nanocomposites. No effect of the previous thermal history was found on the mean relaxation time, evaluated as: $\tau=1 / \omega_{\max }$; where $\omega_{\max }$ is the frequency corresponding to the maximum of the dielectric loss.

Thermal analysis was performed by means of DSC-Q2000 from TA-Instruments calibrated with melting indium. DSC measurements were carried out on a mass of 5-10 mg for all samples. For enthalpy recovery measurements, all experiments began with a heating ramp to a temperature of $T_{g}+30{ }^{\circ} \mathrm{C}$ in order to erase the material's previous thermal history. The samples were subsequently cooled down at a programmed rate of $20{ }^{\circ} \mathrm{C} \mathrm{min}{ }^{-1}$ to reach $T_{g}$ - $80{ }^{\circ} \mathrm{C}$ before being stabilized at the temperature used for annealing in the calorimeter $\left(70{ }^{\circ} \mathrm{C}\right.$ for PS, $80^{\circ} \mathrm{C}$ for PMMA), for times from several minutes to $16 \mathrm{~h}$ before being cooled to $T_{g}-80{ }^{\circ} \mathrm{C}$ at a cooling rate of $20{ }^{\circ} \mathrm{C} \mathrm{min}{ }^{-1}$, and finally reheated at $10{ }^{\circ} \mathrm{C} \mathrm{min}{ }^{-1}$ (see Fig. 2). For the measurements of the enthalpy relaxation at longer aging times $\left(t_{a} \geq 24 \mathrm{~h}\right)$, the annealing of the samples was carried out in an external vacuum oven after erasing of the thermal history and quenching at $20{ }^{\circ} \mathrm{C} \mathrm{min}^{-1}$ of the samples in DSC. After aging, the samples were cooled down again and DSC thermograms were recorded. Second scans were performed immediately after a new quench at $20{ }^{\circ} \mathrm{C} \mathrm{min}^{-1}$. 
As a general procedure in calorimetric experiments, the amount of enthalpy relaxed during aging (and thus recovered during the DSC scan) of a glass for a period of time $t_{a}$ at a given temperature $T_{a}$ was evaluated by integration of the difference between thermograms of aged and unaged samples, according to the equation:

$$
\Delta H\left(T_{a}, t_{a}\right)=\int_{T_{x}}^{T_{y}}\left(C_{p}^{a}(T)-C_{p}{ }^{u}(T)\right) d T
$$

where $C_{p}{ }^{a}(T)$ and $C_{p}{ }^{u}(T)$ are respectively the specific heat of the aged and unaged samples, and $T_{x}$ and $T_{y}$ are respectively temperatures below and above the calorimetric $T_{g}$. For each polymer, $T_{x}$ and $T_{y}$ were chosen as the temperatures at which the unaged and the aged sample scan superimpose below and above $T_{g}$, respectively. Typically, these temperatures were 90 and $140{ }^{\circ} \mathrm{C}$ for PMMA based samples and 95 and $120{ }^{\circ} \mathrm{C}$ for PS based samples.

DSC was also employed for precise characterization of the specific heat of all nanocomposites and the pure polymers. To do so, samples were first heated up at $150{ }^{\circ} \mathrm{C}$ and annealed for $30 \mathrm{~min}$. A sinusoidal temperature modulation of \pm 0.5 every $60 \mathrm{~s}$ was applied to the samples and the specific heat recorded on cooling until $60{ }^{\circ} \mathrm{C}$ at $1{ }^{\circ} \mathrm{C} \mathrm{min}^{-1}$.

\section{Results}

\subsection{BDS characterization}

The normalized dielectric loss of all nanocomposites and the pure polymers is displayed in Fig. 3 as a function of the frequency at two different temperatures. The normalization factors, i.e. the dielectric loss corresponding to the maximum of the peak at the temperatures corresponding to the plots displayed in Fig. 3 are reported in Table 1. In particular the upper and lower panels of the figure respectively present data for PMMA and PS based systems. In the case of PS, the relaxation peak can be straightforwardly attributed to the $\alpha$ process of the polymer. Conversely the upper panel of Fig. 3 is characterized by a relaxation peak, which can be assigned to the merged dielectric response of PMMA $\alpha$ and $\beta$ processes at $150{ }^{\circ} \mathrm{C}$. In the frequency domain the dielectric response related to the $\alpha$ process of PMMA is hardly visible below the temperature of the $\alpha / \beta$ process merging. This is particularly true for the nanocomposites where the $\alpha$ process is masked by the more pronounced conductivity of the samples. However the 

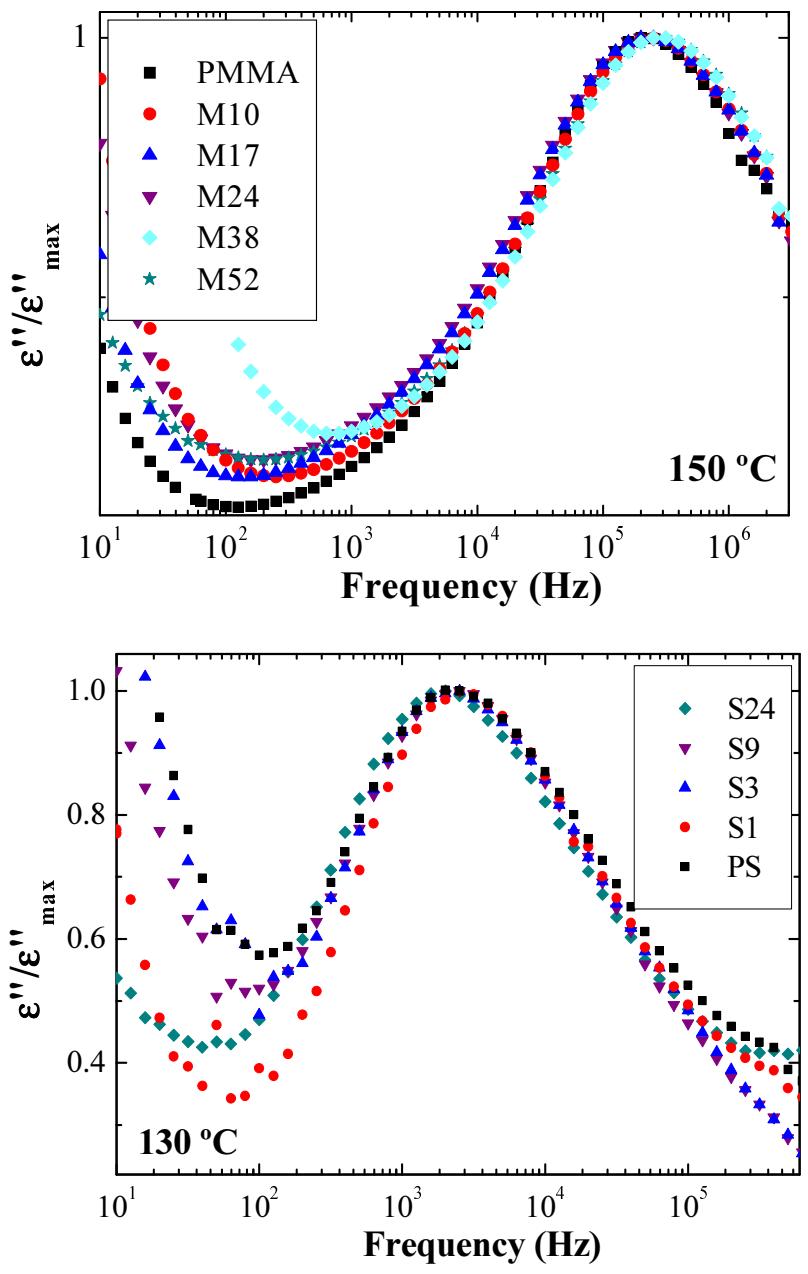

Figure 3: Normalized dielectric loss vs frequency for PMMA (upper panel) and PS (lower panel) based systems. The values of $\epsilon "$ max are reported in Table 1.

representation in the temperature domain reported in Fig. 4 (upper panel) clearly highlights PMMA $\alpha$ process well separated from the $\beta$ process. In the lower panel of the same Fig., PS dielectric response in the temperature domain is reported at $10 \mathrm{~Hz}$. Similarly to Fig. 3, the dielectric loss corresponding to the maximum of the peaks - in the case of PMMA both those of $\alpha$ and $\beta$ processes - are reported in Table 1 . The dielectric response of both pure PMMA and PS is in general agreement with that reported in literature $[23,24]$. From the observation of Figs. 3 and 4 and Table 1, one can conclude 
that PMMA dynamics is not affected by the presence of silica nanoparticles. Except for the sample with the largest silica content (S24), the same can be concluded for PS dynamics. In particular, the characteristic relaxation time and the width of the relaxation of the $\alpha$ and $\beta$ processes are found to be independent of the presence of silica. In the case of PS/silica nanocomposite S24, a slight slowing down of the segmental dynamics in comparison to the other nanocomposites and pure PS can be observed. Furthermore the values of the dielectric loss corresponding to the maximum of the relaxational peak are, within the experimental uncertainty, independent of the silica content and equal to that of the bulk polymers.

Fig. 5 reports the temperature dependence of the characteristic relaxation time of the $\alpha$ process for PS and its nanocomposites, and the $\alpha$ and $\beta$ processes as well as the merged $\alpha / \beta$ process for PMMA and its nanocomposites. As can be seen, except for S24, no effect of the presence of the silica nanoparticles is found in the whole investigated temperature range. The result of unchanged segmental dynamics - observed in the vast majority of the investigated systems - agrees with other results obtained in similar silica nanocomposites [25]. Conversely the slight slowing down of PS dynamics in the nanocomposite $\mathrm{S} 24$ represents a notable exception.

\subsection{Calorimetric characterization}

Fig. 6 displays the temperature dependence of the specific heat for all investigated systems obtained on cooling at $20{ }^{\circ} \mathrm{C} \mathrm{min}{ }^{-1}$ after subtraction of the contribution of silica, by applying the following additive rule: $C_{p, p o l .}(T)=\left(C_{p, t o t .}(T)-w t \%_{S_{i O}} * C_{p, S i O_{2}}(T)\right) / w t \%_{\text {pol. }}$, where $C_{p, t o t .}, C_{p, p o l}$. and $C_{p, S_{2} O_{2}}$ are the total, the polymer and the silica specific heats, respectively, and $w t \%_{\mathrm{SiO}_{2}}$ and $w t \%_{\text {pol. }}$ are the silica and the polymer concentrations, respectively. The specific heat of silica was taken from the literature [26]. The insets of Fig. 6 display the reversing specific heat, obtained on cooling at $1{ }^{\circ} \mathrm{C} \mathrm{min}{ }^{-1}$ with a temperature modulation of $\pm 0.5^{\circ} \mathrm{C}$ every $60 \mathrm{~s}$. These represent the as-measured reversing specific heat (after removal of silica contribution), whereas the specific heat obtained by standard calorimetry have been shifted to superpose the liquid heat capacities of nanocomposites with those of the respective pure polymer. Before proceeding to the analysis of the data, it is worth noting that the reversing specific heats of the pure polymers generally agree with those reported in the ATHAS databank and elsewhere $[27,28]$. Only a slight overestimation of the glassy heat capacity, particularly for PMMA and in all cases smaller than $10 \%$, appears in our data. The first 

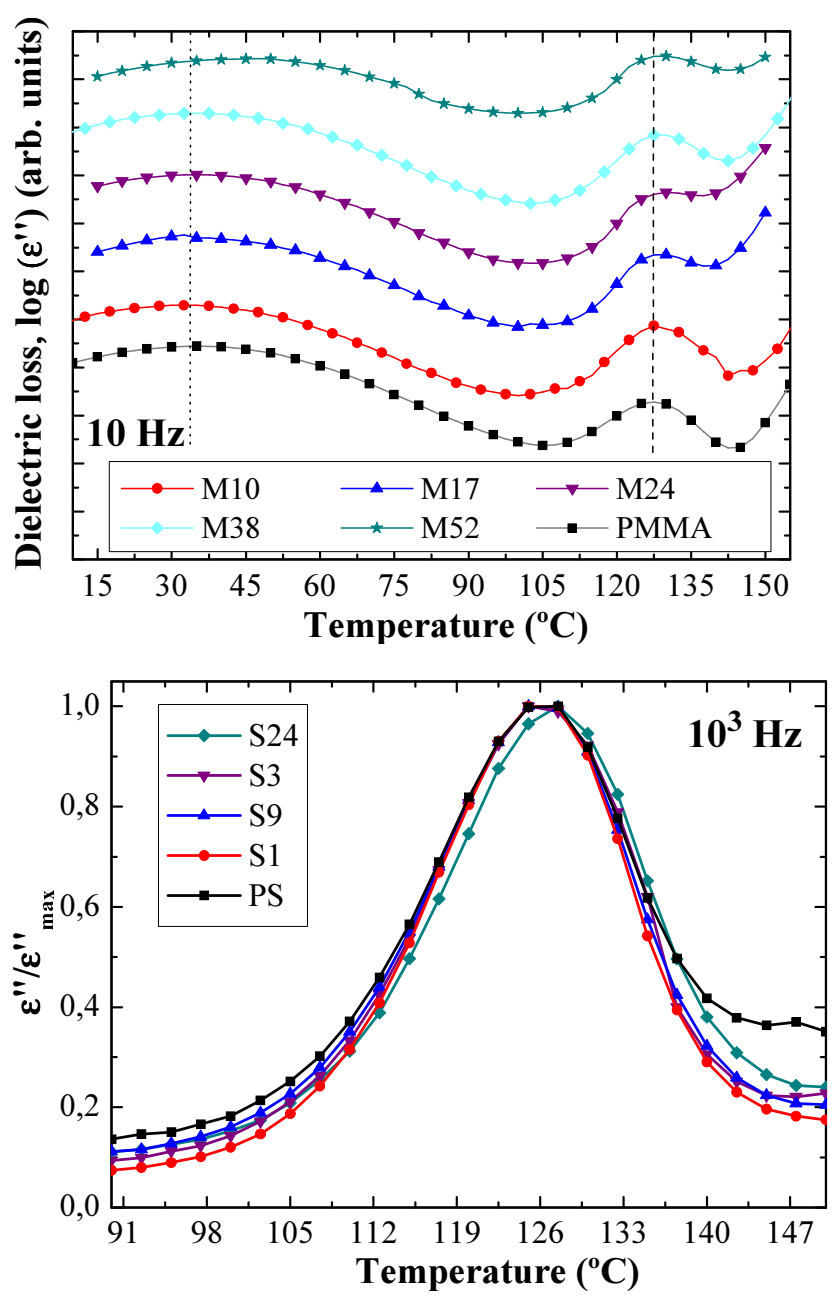

Figure 4: Temperature dependence of the loss part of the dielectric permittivity for: (upper panel) pure PMMA and PMMA/silica nanocomposites at $10 \mathrm{~Hz}$. The dashed and dotted lines respectively indicate the temperature of the $\alpha$ and $\beta$ process; and (lower panel) pure PS and PS/silica nanocomposites at $10^{3} \mathrm{~Hz}$. The values of $\epsilon$ " max are reported in Table 1.

observation that can be done inspecting Fig. 6 is that, within the experimental error, the calorimetric $T_{g}$ - taken as the mid-point temperature of the $C_{p}$ jump (see an example of such determination in Fig. 6, dashed lines in the upper panel) - slightly but systematically decreases with increasing silica content. The PS nanocomposite S24 deviates from this trend, being its $T_{g}$ larger than that of the other PS nanocomposites and practically identical to 

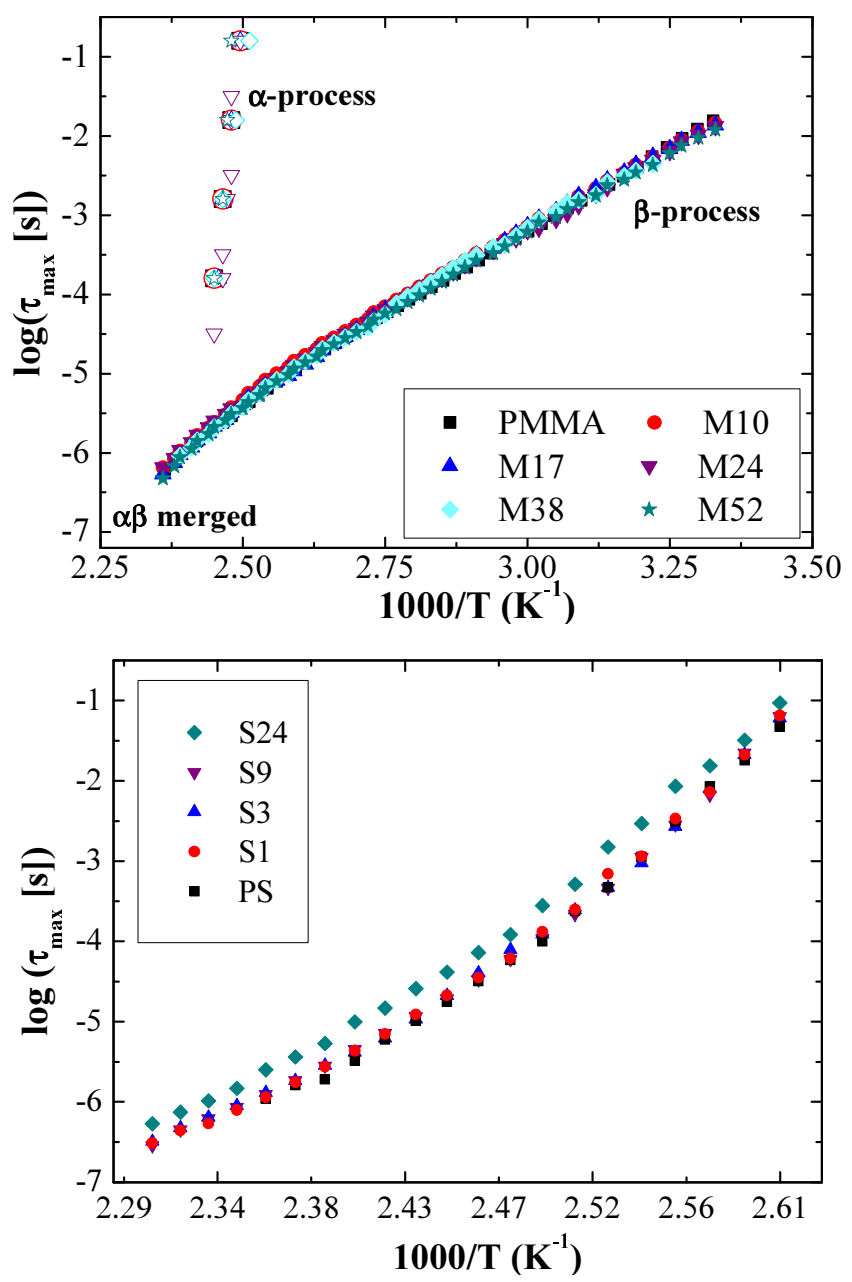

Figure 5: Temperature dependence of the relaxation time for all nanocomposites and pure PMMA and PS as determined isochronally (empty symbols) and isothermally (full symbols).

that of pure PS. This is quantitatively shown in Table 1 , where the $T_{g}$ values of all nanocomposites and the pure polymers are summarized. While $T_{g}$ depression in PS based nanocomposite systems has been reported [10], that in PMMA nanocomposites is in apparent contradiction with the previously reported increase of $T_{g}$ in PMMA/silica nanocomposites [29]. A similar result was reported in poly(ethyl acrylate)/silica nanocomposites [30]. However, it is worth remarking that - differently from the present work - in the case of 
ref. [29], silica nanoparticles are used without any functionalization. This implies that PMMA ester side groups may interact via hydrogen bonding with hydroxyl units on the surface of the silica nanoparticles and provoke a slowing down of PMMA segmental dynamics close to the interface.

The lack of correspondence between the calorimetric $T_{g}$ and the polymer segmental mobility in the investigated nanocomposites is analogous, in the case of PS also quantitatively, to that recently found in polystyrene (PS)/gold nanocomposites [10]. The origin of this apparent discrepancy will be considered in detail in the Discussion section.

Another interesting observation arising from Fig. 6 is that the specific heat jump at $T_{g}$ is reduced in all nanocomposites in comparison to the corresponding pure polymers and such a reduction is more pronounced the larger the silica content. Note that this reduction is an effect related to the polymer matrix, considering that as previously discussed Fig. 6 reports the specific heat values after subtraction of the silica contribution. Furthermore, as suggested by modulated calorimetry results, PMMA and PS melt heat capacities in the nanocomposites are not affected by the presence of nanoparticles and equal to that of the pure polymers. Interestingly the lack of effect on the melt specific heat is accompanied by an unchanged segmental dynamics in the nanocomposites in comparison to the pure polymers. In the case of PMMA nanocomposites, this result apparently contrasts with that found by Sargsyan et al. [31], who - investigating the heat capacity of PMMA/silica nanocomposites - observed a PMMA liquid heat capacity lower in the nanocomposites than in pure PMMA. The discrepancy between the latter results and ours can be easily explained considering that in the work by Sargsyan et al. [31] the specific surface area is considerably larger than in the present work and, similarly to other studies [29], pristine silica nanoparticles are employed in Ref. [31]. Thus, the presence of an immobilized PMMA layer of a couple of nanometers close to the silica surface $[32,31,30,33]$ may play a major role in removing degrees of freedom and this results in an overall decrease of the liquid specific heat. In the case of the present study, the amount of presumably immobilized interface is in all samples negligible and, therefore, no effect on PMMA liquid heat capacity is observed. In the case of PS, to the best of our knowledge, the decrease in the specific heat jump in nanocomposites has not been reported elsewhere.

The previous observations imply that the reduced specific heat jump at $T_{g}$ in the nanocomposites must be attributed to enhanced polymer specific heat in the glassy state of the nanocomposites in comparison to the bulk polymers, 

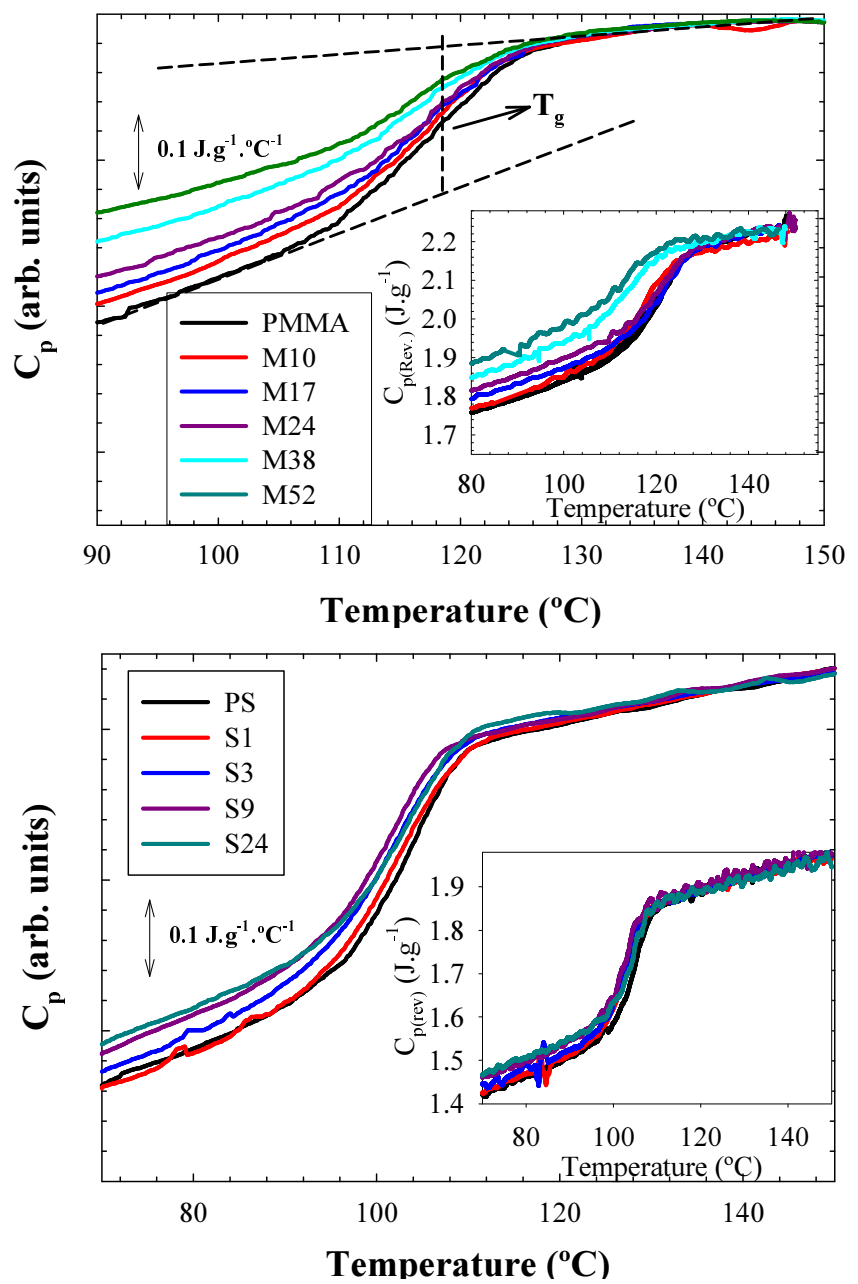

Figure 6: Calorimetric response of all investigated systems obtained on cooling at $20{ }^{\circ} \mathrm{C}$ $\mathrm{min}^{-1}$ for: (upper panel) PMMA based systems; (lower panel) PS based systems. The insets show the calorimetric response of the same systems obtained on cooling at $1{ }^{\circ} \mathrm{C}$ $\min ^{-1}$ with a temperature modulation of $\pm 0.5{ }^{\circ} \mathrm{C}$ every $60 \mathrm{~s}$. All specific heats are normalized to the polymer mass (see text for details). The dashed lines in the upper panel for pure PMMA is an example of how the $T_{g}$ is determined. 
as shown in Fig. 6. The main consequence of this result is that the enthalpy of the glassy polymer in the nanocomposites decreases faster than that of pure PMMA and PS when cooling down below $T_{g}$. This is schematized in Fig. 7, where the temperature dependence of the enthalpy originating from the observed specific heat behaviour is voluntarily emphasized to summarize the complete phenomenology of physical aging for some representative polymer nanocomposite systems. These are: (i) a slight $T_{g}$ depression increasing with the silica content; (ii) a reduction of the $C_{p}$ jump at $T_{g}$, getting larger with the increase of silica content, (iii) a reduction in the total amount of recoverable enthalpy $\Delta H(\infty)$ when increasing silica content in the polymer. The latter result originates from the lower initial enthalpy achieved by the nanocomposites when cooling from above $T_{g}$ to the selected aging temperature. In the nanocomposites this is due to a more rapid decrease of the glassy enthalpy, which may be attributed to a different non-isothermal evolution of the enthalpy among the samples during cooling in the glassy state. It is worth mentioning that, though to a less extent in comparison to the total heat capacity, also the glassy reversing specific heats, measured likewise on cooling and heating, of PS and PMMA in the nanocomposites appear to be systematically larger than those of the pure polymers. Considering that nonisothermal aging below $T_{g}$ is a thermodynamically non-reversible process, the increase in the glassy reversing specific heat in the nanocomposites cannot be directly related to this phenomenon. However, indirect effects of physical aging on the reversing specific heat may be invoked. In particular, considering that this magnitude is a measure of the rate of change of the vibrational degrees of freedom in the glassy state, the larger reversing specific heat may be related to a faster removal of those degrees of freedom when cooling below $T_{g}$.

As will be seen in the next section of the paper, the faster non-isothermal enthalpy evolution of nanocomposites in comparison to the pure polymers is corroborated by isothermal enthalpy recovery results that also show faster evolution towards equilibrium of the thermodynamic state of PMMA and PS in the nanocomposites in comparison to the pure polymers.

\subsection{Enthalpy recovery results}

Figs. 8 and 9 respectively display the specific heat of PMMA in the nanocomposite M24 and that of pure PMMA, and that of PS in the nanocomposite S9 and pure PS as examples, at different aging times, at $80{ }^{\circ} \mathrm{C}$ for PMMA and $70^{\circ} \mathrm{C}$ for PS. Clear differences in the time evolution of the specific 


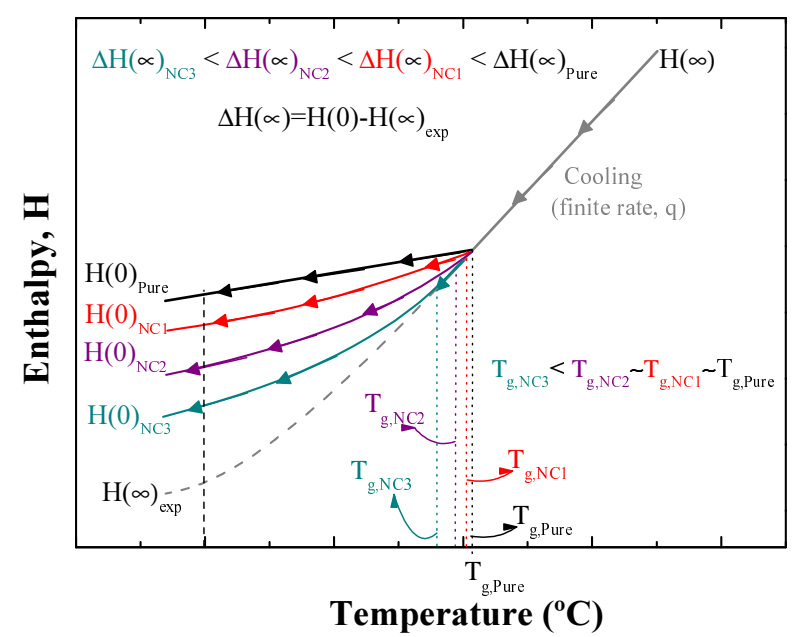

Figure 7: Schematic representation of the temperature dependence of the polymer enthalpy in the nanocomposites and in the pure state when cooling down from above $T_{g}$. The subscripts NC1, NC2 and NC3 refers to nanocomposites with increasing the nanoparticles content. The equilibrium line has been curved to account for the recently observed deviation from the theoretical liquid line [34].

heat line-shape during physical aging between the two displayed nanocomposites on one side and the pure polymers on the other can be noticed. In particular, the following observations can be done: (i) at short aging times (see insets of Figs. 8 and 9) the endothermic overshoot underlying physical aging develops more rapidly in the nanocomposites than in the pure polymers; (ii) at intermediate aging times one can observe that the endothermic overshoot, the typical signature of physical aging, is larger for the nanocomposite; (iii) whereas a plateau in the recovered enthalpy seems to appear after about $25 \mathrm{hrs}$ aging time for M24 (though with an evolving shape of the specific heat versus temperature curves), and after $12 \mathrm{hrs}$ aging time for S9, the endothermic overshoot observed in the pure polymers still evolves in intensity after aging times larger than $100 \mathrm{hrs}$. Similar results have been found for the other nanocomposites.

From the specific heat versus temperature plots, the recovered enthalpy as a function of the aging time can be obtained through eq 1. This is shown in Fig. 10 for all investigated samples. A preliminary observation of the Fig. indicates that a plateau in the log time scale is reached for all investigated samples. Despite this, the recovered enthalpy at this plateau is never larger 

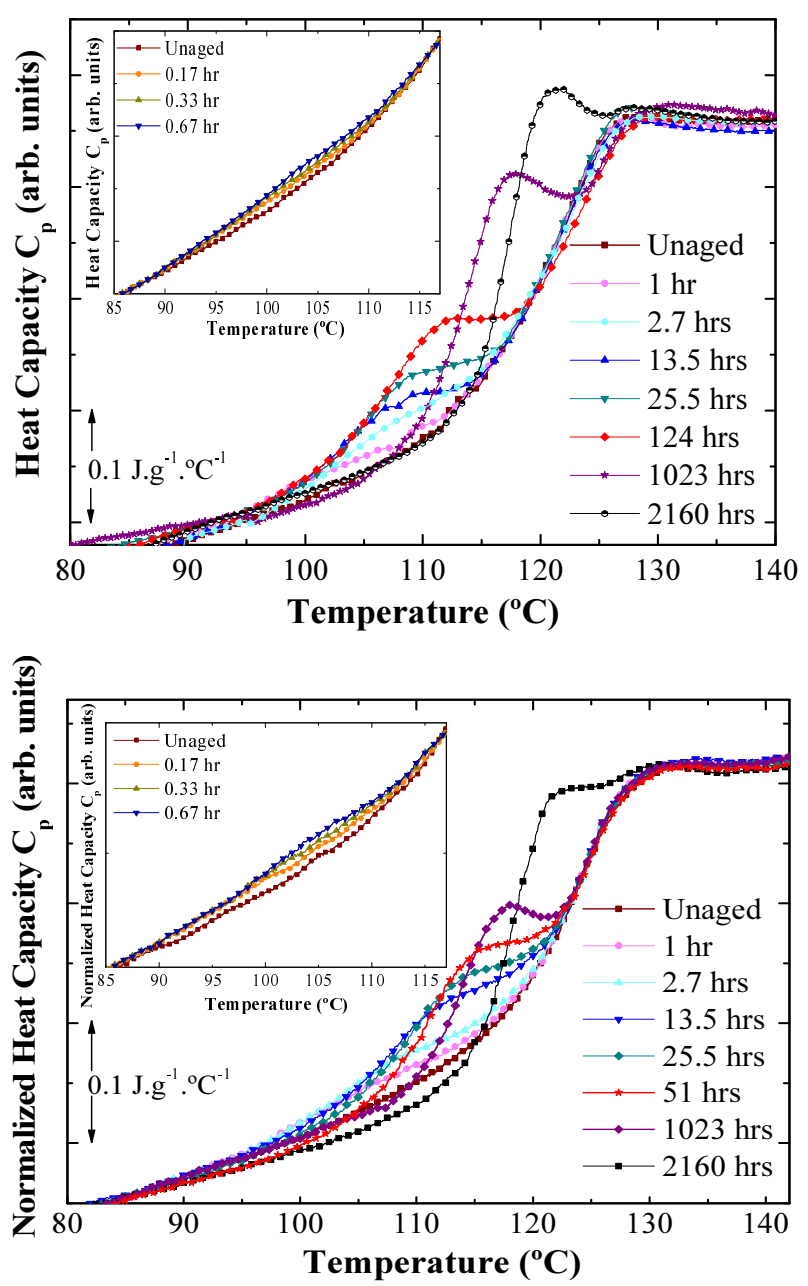

Figure 8: Calorimetric response of pure PMMA (upper panel); and the nanocomposite M24 (lower panel) at different aging times at $80{ }^{\circ} \mathrm{C}$ after cooling at $20{ }^{\circ} \mathrm{C}$ min ${ }^{-1}$ from above $T_{g}$. The insets display the same results but at relatively short aging times (shorter than $40 \mathrm{~min}$ ). 

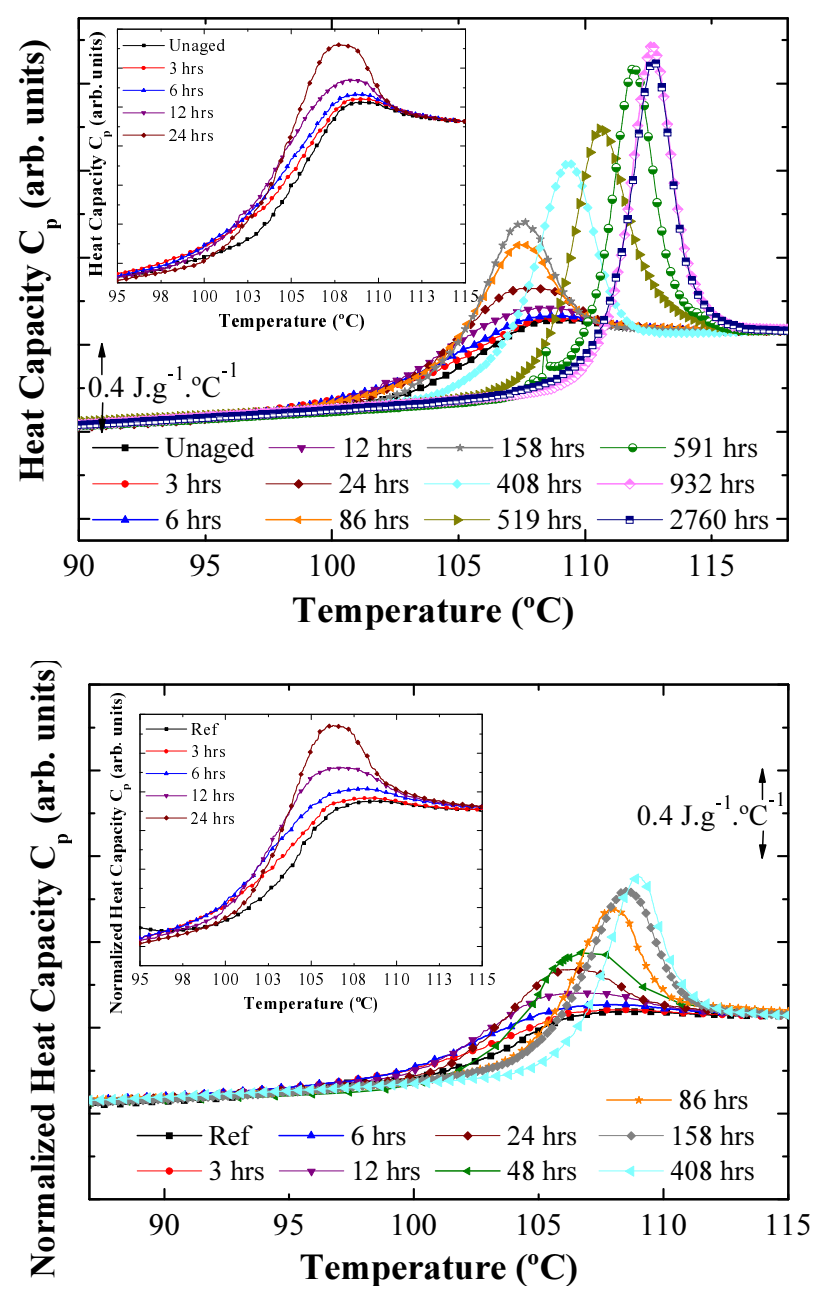

Figure 9: Calorimetric response of pure PS (upper panel); and the nanocomposite S9 (lower panel) at different aging times at $70{ }^{\circ} \mathrm{C}$ after cooling at $20{ }^{\circ} \mathrm{C}$ min ${ }^{-1}$ from above $T_{g}$. The insets display the same results but at relatively short aging time (shorter than 12 hrs). 
than 2.1 $\mathrm{J} \mathrm{g}^{-1}$ for PMMA and $2.4 \mathrm{~J} \mathrm{~g}^{-1}$ for PS, which is only a fraction of the enthalpy that would be recovered if the enthalpy of the equilibrium melt was extrapolated at $80{ }^{\circ} \mathrm{C}$ for PMMA and $70^{\circ} \mathrm{C}$ for PS. This issue has been discussed in detail in a recent work by us [34] and is in agreement with previous results on PMMA/silica nanocomposites [8], PS/gold nanocomposites [10] and several studies on a number of polymer glasses [35, 36, 37, 38].

As far as the differences in the enthalpy recovery trend between the pure polymers, and their nanocomposites is concerned, inspection of Fig. 10 indicates the following results: (i) the total recovered enthalpy, namely that of the plateau, decreases with increasing silica content; (ii) at short aging times the amount of recovered enthalpy is larger in the case of nanocomposites; (iii) the time needed to reach the plateau gets shorter with increasing silica content. A special case is that of PS/silica nanocomposites S24 displaying a time to reach the plateau similar to the nanocomposite S9 but with a smaller total recoverable enthalpy.

The shorter time to reach the plateau generally displayed by the nanocomposites indicates that the enthalpy recovery of nanocomposites is generally faster than that of the pure polymers, in agreement with recent results obtained on another set of PMMA nanocomposites with silica particles of different sizes and obtained under different preparation conditions [7, 8], and those relative to PS/gold nanocomposites [10]. To explain the significant reduction of the total recovered enthalpy in the investigated nanocomposites, it is worth noticing that - as a result of the larger specific heat in the glassy state (see Fig. 6) - the enthalpies of PMMA and PS in the nanocomposites at the beginning of the physical aging process are lower than those of the pure polymers (see Fig. 7). Thus we can qualitatively attribute the reduction in the total recovered enthalpy to the non-isothermal aging occurring during cooling below $T_{g}$ and subsequent reheating to obtain the reference curve as described in the Experimental section. This conclusion is also supported by the shape of the enthalpy recovery curves as a function of the aging time. Indeed, fully developed recovery curves normally present a sigmoidal shape as in the case of pure PMMA and PS of the present study, whereas in nanocomposites with large silica contents the short aging times part of the aging curves is missing.

The experimental data concerning the enthalpy recovery of PMMA and PS in all samples at the aging temperature of respectively $80{ }^{\circ} \mathrm{C}$ and $70{ }^{\circ} \mathrm{C}$ are displayed in a normalized representation as a function of the aging time in Fig. 11. In this case, data are reported as relaxation isotherms, defined 

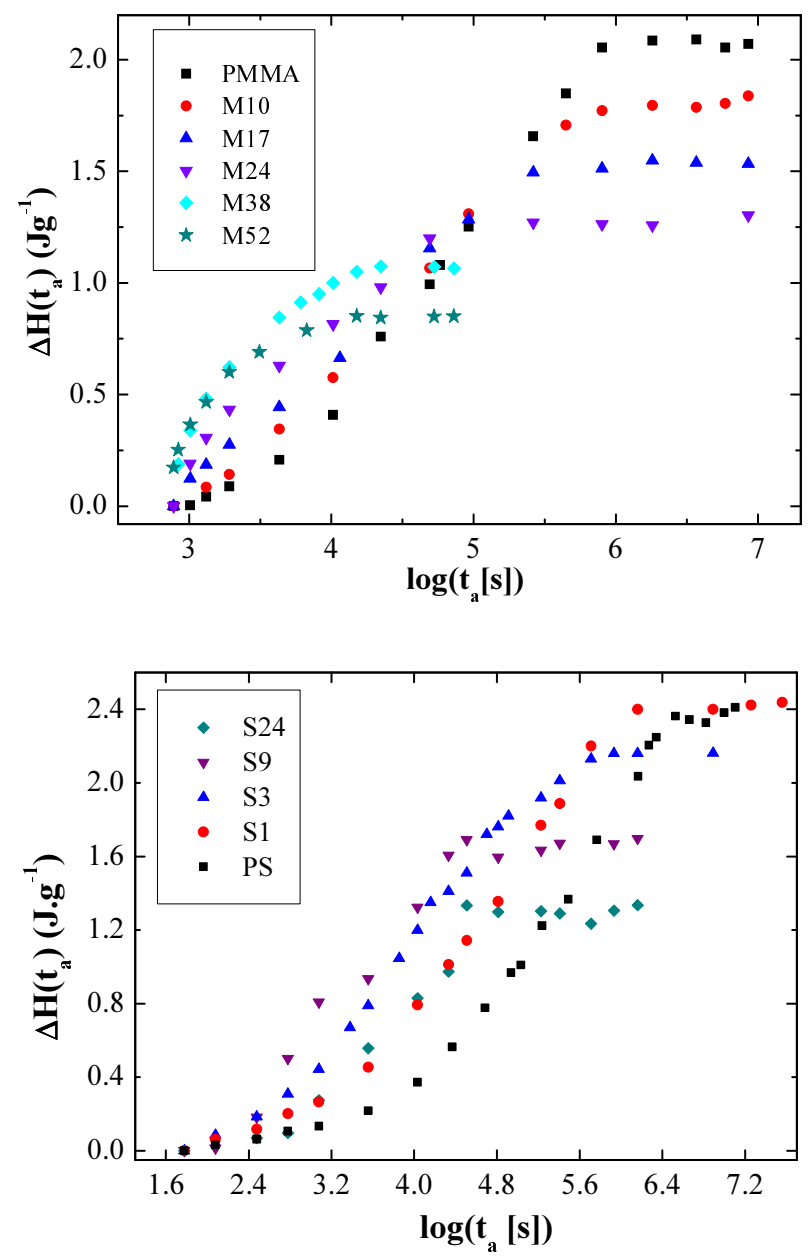

Figure 10: Recovered enthalpy as a function of aging time for: (upper panel) PMMA based systems at $80{ }^{\circ} \mathrm{C}$; and (lower panel) PS based systems at $70^{\circ} \mathrm{C}$. 
by the widely used function describing enthalpy relaxation of glasses:

$$
\bar{\phi}_{H}(t)=\frac{H(t)-H_{\infty}}{H_{0}-H_{\infty}}
$$

where the value of the enthalpy at zero aging time related to pure PMMA and PS was employed for $H_{0}$, since these systems display a negligible nonisothermal aging during the cooling procedure preceding the isothermal enthalpy recovery process. $H_{\infty}$ is the long term value of the enthalpy, namely that corresponding to the experimental plateau in the enthalpy versus log time plots. This representation highlights the difference in the time to recover equilibrium and the similar slope of the relaxation function of all systems at the point of its maximum variation.

To conclude this section, we emphasize the systematic alteration of the enthalpy recovery trend - in comparison to the pure polymers - occurring when increasing silica content in the polymers. In particular, increasing silica content provokes a general acceleration of the physical aging process, which results in an apparently smaller recovered enthalpy in the nanocomposites as a consequence of the enthalpy recovery during non-isothermal physical aging occurring in proximity of $T_{g}$.

\section{Discussion}

In the previous section of the paper, we have seen that in the investigated nanocomposites the amount of silica particles, and hence the specific surface area, has no appreciable effects on the polymer segmental dynamics, except for PS/silica nanocomposite S24. Even if some changes of the segmental mobility are present in a layer of several nanometers close to the interface with silica - as suggested by several studies $[30,32]$-, this can be considered to be negligible, since the typical interparticle distance is always larger than 100 $\mathrm{nm}$ in the investigated nanocomposites. The possibly altered dynamics at the interface is certainly not sufficient to justify the significant acceleration of physical aging found in the present study. Thus, a suitable framework to rationalize the acceleration of physical aging in the investigated nanocomposites is the free volume holes diffusion model, first presented by Curro et al. [18] to describe the volume recovery in glassy poly(vinyl acetate) (PVAc) and later employed to rationalize the accelerated physical aging in thin films $[11,12,19,16,17]$ and polymer nanocomposites [7, 8]. According to this model a nanostrucutured system can age faster than the bulk counterpart, 

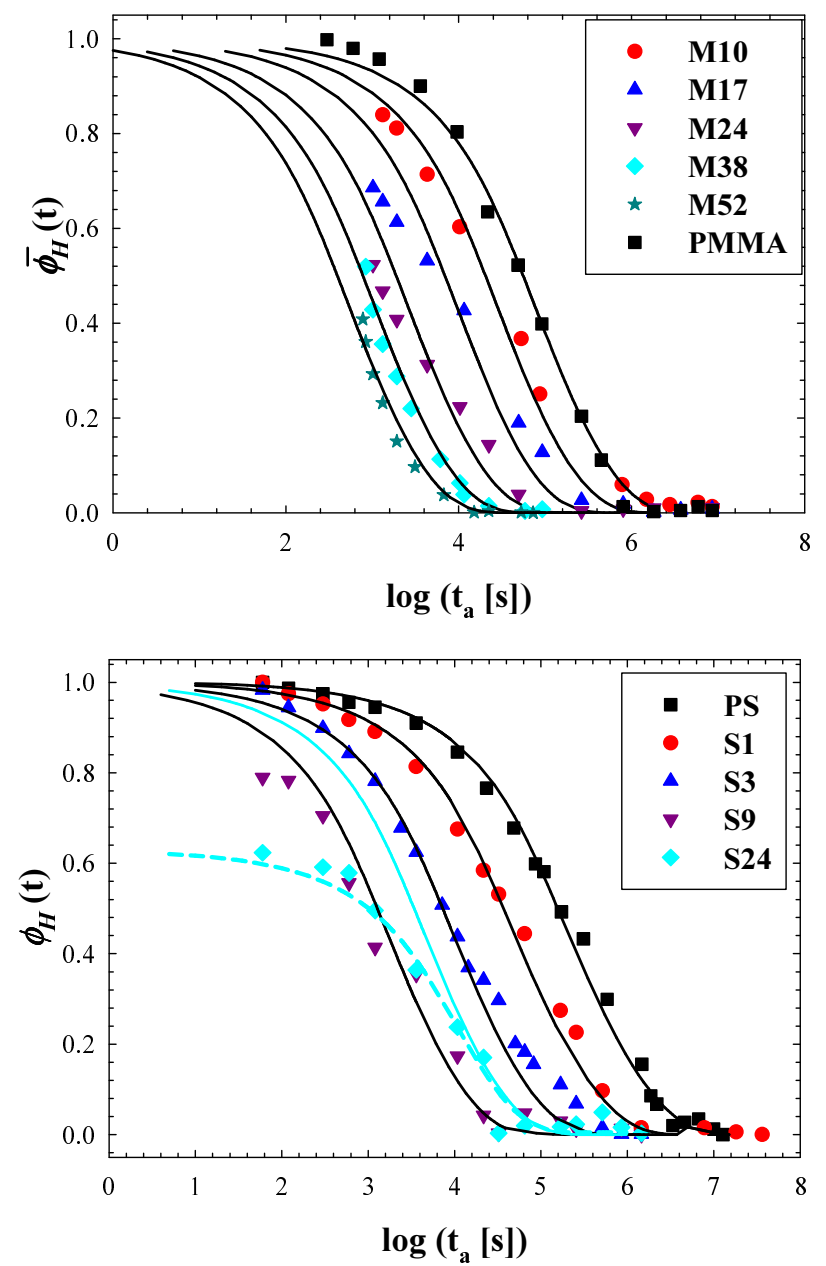

Figure 11: Structural relaxation function as a function of aging time for: (upper panel) PMMA based systems at $80^{\circ} \mathrm{C}$; and (lower panel) PS based systems at $70^{\circ} \mathrm{C}$. Continuous lines correspond to the fits of the diffusion model to experimental data. To highlight the fact that a diffusion coefficient smaller than that used for the other PS nanocomposites was employed for S24, the fit to the diffusion model of this nanocomposite was indicated with a light blue continuous line. The dashed line is the fit of the diffusion to S24 data assuming a lower recoverable enthalpy (see text for details). 
with no need to invoke enhancement of the polymer segmental dynamics. A detailed development of the model for polymer nanocomposites can be found elsewhere [22]. Here we briefly summarize the main aspects of the model. According to this model, the spontaneous densification of an outof-equilibrium glass occurs via the diffusion of free volume holes and their elimination at the polymer surface, in the case of present study the interface polymer-nanoparticles. Thus physical aging can be treated as a simple Fickian diffusion problem, which can be solved in two dimensions [22]. In this case the diffusion problem can be solved in equivalent films with thicknesses directly related to the area of silica to volume of polymer in the nanocomposites by: $h=2 V / A$. The main equation describing the space-time evolution of the free volume fraction $f_{v}(x, t)$ is:

$$
\frac{\partial f_{v}(x, t)}{\partial t}=\frac{\partial}{\partial x}\left(D \frac{\partial f_{v}(x, t)}{\partial x}\right)
$$

where $D$ is the diffusion coefficient of free volume holes and $t$ and $x$ are respectively the temporal and spatial coordinates. The diffusion coefficient depends on the thermodynamic state of the glass and can be related to the free volume fraction by the Doolittle equation [39]:

$$
D=D_{\infty} \exp \left(\frac{B}{f_{v \infty}}-\frac{B}{f_{v}(x, t)}\right)
$$

where $D_{\infty}$ and $B$ are material specific parameters, being $D_{\infty}$ also dependent on the temperature, and $f_{v \infty}$ is the free volume fraction at the beginning of the physical aging process. Eq 3, in combination with eq 4, can be solved numerically imposing the appropriate boundary conditions [22].

The equivalent thickness of the sample, $h$, was evaluated as the inverse of the total of the area to volume ratio. The latter is the sum of the external area to volume ratio related to the nanoparticles content, and the internal one [22]. The latter corresponds to an ill-defined intrinsic internal length scale of polymers namely that able to provide the fitting of pure PMMA and PS recovery data [18]. The existence of an internal length scale is so far not proved though theorized [18]. Alternative approaches rather employ lattice contraction to account for the presence of physical aging also in bulk glasses [19]. Thus the equivalent thickness can be calculated as follows:

$$
h=\frac{h_{i n t} h_{e x t}}{h_{\text {ext }}+h_{\text {int }}}
$$


where $h_{\text {int }}$ and $h_{\text {ext }}$ are respectively related to the internal and the external surfaces.

$f_{v 0}$ and $f_{v \infty}$, respectively the unrelaxed and relaxed free volume fractions, can be determined employing the Simha-Somcynski equation of state (EOS) theory [40]. Details on the application of this theory can be found elsewhere $[22,10]$. Here we just report the values of the free volume fractions: $f_{v 0}=$ 0.093 and $f_{v \infty}=0.072$ for PMMA and $f_{v 0}=0.083$ and $f_{v \infty}=0.061$ for PS.

The parameters $B$ and $D_{\infty}$ of the Doolittle equation and PMMA internal length scale $h_{\text {int }}$ were previously determined in PMMA with silica and PS with gold nanocomposites displaying homogeneous dispersion of nanoparticles $[22,10]$. These are: $B=0.715, D_{\infty}=6.7 \times 10^{-14} \mathrm{~cm}^{2} \mathrm{~s}^{-1}$ and $h_{\text {int }}=2000$ nm for PMMA; and $B=0.64, D_{\infty}=2.5 \times 10^{-14} \mathrm{~cm}^{2} \mathrm{~s}^{-1}$ and $h_{\text {int }}=8000$ nm for PS. ${ }^{1}$ Considering the PS/silica nanocomposite S24 possesses a segmental mobility about 2.5 times slower than the other PS nanocomposites (at least in the neighbourhood of $T_{g}$ ), a value of $D_{\infty}=9.3 \times 10^{-15} \mathrm{~cm}^{2} \mathrm{~s}^{-1}$ was employed for this nanocomposite. These parameters were introduced as inputs when fitting the data to the diffusion model. The fits of the free volume diffusion model with the equivalent thickness $h$ as the only fitting parameter are reported in Fig. 11. As can be observed, the quality of the fits is generally good, apart from that of S24 (continuous light blue line). In this case, the diffusion model fails to catch the initial evolution of the enthalpy recovery process. However, it is worth mentioning that the diffusion model is developed to describe the enthalpy recovery process after a perfect quench, whereas our experimental cooling rate is $20{ }^{\circ} \mathrm{C} \mathrm{min}^{-1}$. Thus, the effect of non-isothermal aging during cooling from above $T_{g}$ - particularly important for samples with high silica content - is not taken into account by the so-developed model. If a smaller recoverable enthalpy - corresponding to physical aging performed after a quench at a finite rate - is introduced as input in the diffusion model, the recovered enthalpy data after quenching at 20 ${ }^{\circ} \mathrm{C} \min ^{-1}$ can be accurately described as shown by the dashed light blue line in Fig. 11b. The values of the fitted equivalent thicknesses are displayed in Table 1. It is worth noticing that all PS/silica nanocomposites, except S24, display an equivalent thickness corresponding to the one evaluated assuming no aggregation of silica nanoparticles (calculated as: $h_{e x t}=d\left((\pi / 6 v)^{2 / 3}-1\right)$,

\footnotetext{
${ }^{1}$ An inaccurate value was reported in Ref. [10] $\left(h_{\text {int }}=6600 \mathrm{~nm}\right)$. In the present work we report the corrected value.
} 
where $d$ is the nanoparticle diameter and $v$ the nanoparticle volume fraction in the nanocomposite), whereas the values of the equivalent thickness of PMMA/silica nanocomposites are systematically larger than the calculated ones. We can interpret these results considering the lower degree of aggregation in PS based nanocomposites in comparison to PMMA based ones, as qualitatively suggested by TEM micrographs (see Fig. 1). Another interpretation emerges from the fact that silanization of silica does not imply the full recovery of silica nanoparticles. This means that those sites at the interface where hydrogen bonding is possible between PMMA and silica would not be available for diffusing free volume holes out of the polymer matrix, thus reducing the effective specific surface area in these systems, the theoretical being overestimated.

The ability of the free volume holes diffusion model to describe enthalpy recovery data, in combination with the lack of dependence of the segmental mobility on the silica content shown by BDS, indicates that any explanation of the out-of-equilibrium dynamics only in terms of the intrinsic molecular mobility of the glass must be revisited. Interestingly this consideration is also supported by the behaviour of PS/silica nanocomposite S24, displaying the following behaviour: (i) slowing down of the segmental dynamics in comparison to pure PS; (ii) calorimetric $T_{g}$ equal to that of pure PS; (iii) glassy heat capacity larger than that of pure PS; and (iv) faster physical aging in comparison to pure PS but slower in comparison to that of the nanocomposite with smaller nanoparticles content (S9).

An interesting feature of the dependence of enthalpy recovery on silica specific surface area is that the larger this quantity, the smaller the total apparent recovered enthalpy. As already commented, this can be understood as due to the lower initial enthalpy state reached by the nanocomposites as a result of non-isothermal aging occurring when cooling down, which can be significant particularly in proximity of the $T_{g}$. In the present study, for the lowest equivalent thickness of around $170 \mathrm{~nm}$ for PMMA and $450 \mathrm{~nm}$ for PS - obtained from the fitting to the diffusion model -, the recoverable enthalpy decreases to more than $60 \%$ in comparison to pure PMMA and about $50 \%$ for PS. Thus, if no other factor interferes when increasing the specific surface area, at a sufficiently small equivalent thickness, the diffusion of free volume holes model envisages an evolution of the recovered enthalpy so fast that conventional experimental techniques are going to fail in capturing such evolution. This can be at least one reason for the observation of the absence of physical aging effects $[41,42,29]$ or the reduction of this process 
$[43,41,44,45,46,15,47,48]$ in a number of nanostructured glasses with a specific surface area corresponding to an equivalent thickness smaller than $100 \mathrm{~nm}$. This idea is corroborated by recent permeability determination during physical aging in thin films of glassy polysulfone [15]. In such work the permeability of ultrathin polymer films at the beginning of the aging process is significantly lower than in the corresponding bulk polymer. This result well agrees with the interpretation that significant densification has occurred during the cooling and the non-isothermal aging before temperature stabilization and data collection. Furthermore, a very recent study on the physical aging of several molecular glass formers with thickness smaller than $100 \mathrm{~nm}$ studied by an equipment allowing for fast thermal equilibration (cooling rate of about $10^{4}-10^{5}{ }^{\circ} \mathrm{C} \mathrm{s}^{-1}$ ), actually provides evidence for accelerated physical aging at such small thickness [49], as predicted by the diffusion model.

Nonetheless it is worth mentioning that, at specific surface areas corresponding to equivalent thicknesses shorter than about $100 \mathrm{~nm}$, a nonnegligible effect of the molecular mobility close to the interface may be of some importance. This is particularly important in those nanostructured systems presenting strong polymer/substrate interactions [33, 44, 45, 42, 29, $31,46,32]$. In this case, the resulting alteration of the molecular mobility may significantly affect the diffusion coefficient of free volume holes. Thus the resulting rate of physical aging will be the result of two, often competing, factors: the amount of interface and the polymer molecular mobility. In the present study, the compensation between these two factors is highlighted by the aging behaviour of PS/silica nanocomposites S24. This presents slower segmental mobility in comparison to the other PS nanocomposites and the pure polymer. Thus the acceleration of physical aging is not as pronounced as it would be if the mobility was unaffected by the presence of silica nanoparticles.

Another interesting observation can be done comparing the aging behaviour of PS/silica nanocomposites of this study with previously reported enthalpy recovery data on PS/gold nanocomposites [10]. In particular, comparing the enthalpy recovery behaviour of the nanocomposite S1 of this study with PS/gold nanocomposite with $5 \%$ gold nanoparticles with a diameter of $60 \mathrm{~nm}$ (see Fig. 10 of Ref. [10]), one can put forward the following observation: (i) the nanoparticles specific surface area in the two nanocomposites is equal; (ii) the two nanocomposites present the same evolution of the enthalpy during physical aging. These two observations allow to conclude that, at least for not too small equivalent thicknesses, the nature of the dispersed 
nanoparticles is of no importance, whereas the nanoparticles surface area is of paramount importance in determining the rate of physical aging. This conclusion generally agrees with the idea that diffusion of free volume holes controls the rate of physical aging in nanostructured systems.

Apart from the acceleration of physical aging in PMMA and PS nanocomposites with silica in comparison to the pure polymers, another outcome of our study is the observed $T_{g}$ depression (see Fig. 6 and Table 1 ). The same arguments based on the diffusion of free volume holes can be put forward to explain these effects [10]. In particular, given a concentration independent polymer segmental dynamics, the glass transition is expected to occur at lower temperatures in systems where free volume holes have to travel a shorter distance to diffuse to the interface, thus maintaining the thermodynamic equilibrium at lower temperatures. In PMMA/silica nanocomposites of the present study, this effect of $T_{g}$ depression is experimentally visible at relatively large silica contents, whereas even PS nanocomposites with the lowest silica concentration display experimentally significant $T_{g}$ depression, in agreement with the results recently found in PS/gold nanocomposites [22]. This is consistent with literature results showing stronger effects of $T_{g}$ depression in PS thin films than in other polymers, such as PMMA [50], and the fact that accelerated physical aging is found in PS nanocomposites at equivalent thicknesses considerably larger than PMMA nanocomposites.

\section{Conclusions}

The effect of silica content on the segmental mobility, calorimetric $T_{g}$, and enthalpy relaxation of PMMA and PS was investigated. The segmental mobility of PMMA and PS (with only one exception), as assessed by BDS, was generally found to be insignificantly modified by the presence of silica particles in the polymer, in a very wide range of silica contents. Conversely, the calorimetric $T_{g}$ in those systems presenting segmental dynamics equal to that of the bulk polymer was observed to decrease. The latter observation puts in evidence the existence of a decoupling between the segmental mobility as probed by BDS, and calorimetric $T_{g}$ measurements. Furthermore, in disagreement with the well anchored idea that this process is only related to the materials segmental mobility, the physical aging process of PMMA and PS in silica nanocomposites, measured by means of DSC, was found to be deeply altered by the presence of silica particles. The systematic acceleration of the physical aging process with increasing silica particles content, i.e. the 
specific surface area, was proved in a wide range of concentrations, for which PMMA and PS segmental mobility remained unchanged.

The present study allowed a deep understanding of the physical aging process in polymer nanocomposites, by evidencing that the acceleration of the physical aging process is systematically accompanied by a decrease of the heat capacity jump, a reduction in the amount of recoverable enthalpy, and a depression of the calorimetric $T_{g}$. Particularly, it was demonstrated that the faster physical aging of PMMA and PS in the silica nanocomposites is responsible for the different systematic effects of silica content on PMMA and PS thermodynamic characteristics. Finally, the speeding up of PMMA and PS physical aging, as well as the alteration of other PMMA and PS thermodynamic properties in presence of silica nanoparticles, were rationalized by a model based on the diffusion of free volume holes towards the polymer/silica interface. It was shown that the free volume holes diffusion model is able to quantitatively describe the physical aging behaviour of PMMA and PS in the nanocomposites, and to explain both the decoupling between PMMA and PS segmental mobility and rate of physical aging, and that between the segmental mobility as probed by BDS and the calorimetric $T_{g}$.

\section{ACKNOWLEDGEMENTS}

The authors acknowledge the University of the Basque Country and Basque Country Government (Ref. No. IT-436-07, Depto. Educación, Universidades e Investigación and Spanish Minister of Education (Grant No. MAT 2007-63681) for their support. The SGIker UPV/EHU is acknowledged for the electron microscopy facilities of the Gipuzkoa unit. Energy Strategy Associates Inc. is acknowledged for kindly providing the silica nanoparticles.

\section{References}

[1] F. Hussain, M. Hojjati, M. Okamoto, R. E. Gorga, J. Comp. Mat. 40 (2006) 1511-1575.

[2] D. Blond, V. Barron, M. Ruether, K. P. Ryan, V. Nicolosi, W. J. Blau, J. N. Coleman, Adv. Funct. Mat. 16 (2006) 1608-1614.

[3] R. D. Priestley, Soft Matter 5 (2009) 919-926.

[4] A. Kovacs, J. Pol. Sci. 30 (1958) 131-147. 
[5] L. C. E. Struik, Physical Aging in Amorphous Glassy Polymers and Other Materials, Elsevier Science, Amsterdam, The Netherlands, 1 edition, 1978.

[6] T. Hecksher, N. Olsen, K. Niss, J. Dyre, J. Chem. Phys. 133 (2010) 174514.

[7] V. M. Boucher, D. Cangialosi, A. Alegría, J. Colmenero, J. GonzálezIrun, L. M. Liz-Marzan, Soft Matter 6 (2010) 3306-3317.

[8] V. M. Boucher, D. Cangialosi, A. Alegría, J. Colmenero, Macromolecules 43 (2010) 7594-7603.

[9] V. M. Boucher, D. Cangialosi, A. Alegría, J. Colmenero, J. Non-Cryst. Sol. 357 (2011) 605-609.

[10] V. M. Boucher, D. Cangialosi, A. Alegría, J. Colmenero, I. PastorizaSantos, L. M. Liz-Marzan, Soft Matter 7 (2011) 3607-3620.

[11] P. H. Pfromm, W. J. Koros, Polymer 36 (1995) 2379-2387.

[12] K. D. Dorkenoo, P. H. Pfromm, Macromolecules 33 (2000) 3747-3751.

[13] M. S. McCaig, D. R. Paul, Polymer 41 (2000) 629-637.

[14] Y. Huang, D. R. Paul, Macromolecules 38 (2005) 10148-10154.

[15] B. W. Rowe, B. D. Freeman, D. R. Paul, Polymer 50 (2009) 5565-5575.

[16] D. Cangialosi, M. Wubbenhorst, J. Groenewold, E. Mendes, H. Schut, A. van Veen, S. J. Picken, Phys. Rev. B 70 (2004) 224213.

[17] D. Cangialosi, M. Wubbenhorst, J. Groenewold, E. Mendes, S. J. Picken, J. Non-Cryst. Solids 351 (2005) 2605-2610.

[18] J. G. Curro, R. R. Lagasse, R. Simha, Macromolecules 15 (1982) 16211626.

[19] M. S. McCaig, D. R. Paul, J. W. Barlow, Polymer 41 (2000) 639-648.

[20] A. W. Thornton, K. M. Nairn, A. J. Hill, J. M. Hill, Y. Huang, J. Membr. Sci. 338 (2009) 38-42. 
[21] A. W. Thornton, A. J. Hill, Ind. Eng. Chem. Res. 49 (2010) 1211912124.

[22] D. Cangialosi, V. M. Boucher, A. Alegra, J. Colmenero, J. Chem. Phys. 135 (2011) 014901.

[23] R. Bergman, F. Alvarez, A. Alegria, J. Colmenero, J. Chem. Phys. 109 (1998) 7546-7555.

[24] G. A. Schwartz, J. Colmenero, A. Alegria, J. Non-Cryst. Solids 353 (2007) 4298-4302.

[25] R. B. Bogoslovov, C. M. Roland, A. R. Ellis, A. M. Randall, C. G. Robertson, Macromolecules 41 (2008) 1289-1296.

[26] J. Holm, O. Kleppa, E. Westrum, Geochim. Cosmochim. Acta 31 (1967) 2289-2307.

[27] B. Wunderlich, M. Pyda, http://athas.prz.edu.pl/ (2005-2011).

[28] A. Soldera, N. Metatla, A. Beaudoin, S. Said, Y. Grohens, Polymer 51 (2010) 2106-2111.

[29] R. D. Priestley, P. Rittigstein, L. J. Broadbelt, K. Fukao, J. M. Torkelson, J. Phys.: Condens. Matter 19 (2007) 205120.

[30] J. Berriot, H. Montes, F. Lequeux, D. Long, P. Sotta, Macromolecules 35 (2002) 9756-9762.

[31] A. Sargsyan, A. Tonoyan, S. Davtyan, C. Schick, Eur. Pol. J. 43 (2007) 3113-3127.

[32] S. E. Harton, S. K. Kumar, H. Yang, T. Koga, K. Hicks, E. Lee, J. Mijovic, M. Liu, R. S. Vallery, D. W. Gidley, Macromolecules 43 (2010) 3415-3421.

[33] D. Fragiadakis, P. Pissis, J. Non-Cryst. Sol. 353 (2007) 47-51.

[34] V. M. Boucher, D. Cangialosi, A. Alegria, J. Colmenero, Macromolecules 44 (2011) 8333-8342.

[35] J. Cowie, S. Harris, I. McEwen, J. Polym. Sci. Pt. B-Polym. Phys. 35 (1997) 1107-1116. 
[36] J. Duenas, A. Garayo, F. Colomer, J. Estelles, J. Ribelles, M. Pradas, J. Polym. Sci. Pt. B-Polym. Phys. 35 (1997) 2201-2217.

[37] J. Hutchinson, P. Kumar, Thermochim. Acta 391 (2002) 197-217.

[38] L. Andreozzi, M. Faetti, M. Giordano, F. Zulli, Macromolecules 38 (2005) 6056-6067.

[39] A. K. Doolittle, J. Appl. Phys. 22 (1951) 1471-1475.

[40] R. Simha, T. Somchynski, Macromolecules 2 (1969) 342-350.

[41] S. Kawana, R. A. L. Jones, Eur. Phys. J. E 10 (2003) 223-230.

[42] R. D. Priestley, L. J. Broadbelt, J. M. Torkelson, Macromolecules 38 (2005) 654-657.

[43] S. L. Simon, J. Y. Park, G. B. McKenna, Eur. Phys. J. E 8 (2002) 209-216.

[44] H. B. Lu, S. Nutt, Macromolecules 36 (2003) 4010-4016.

[45] H. B. Lu, S. Nutt, Mac. Chem. Phys. 204 (2003) 1832-1841.

[46] S. Amanuel, , A. N. Gaudette, S. S. Sternstein, J. Polym. Sci., Polym. Phys. Ed. 46 (2008) 2733-2740.

[47] A. Y. H. Liu, J. Rottler, J. Polym. Sci. Pt. B-Polym. Phys. 47 (2009) 1789-1798.

[48] J. E. Pye, K. A. Rohald, E. A. Baker, C. B. Roth, Macromolecules 43 (2010) 8296-8303.

[49] A. Sepulveda, E. Leon-Gutierrez, M. T. Clavaguera-Mora, J. ViejoRodriguez, Phys. Rev. Lett 107 (2011) 025901.

[50] J. A. Forrest, K. Dalnoki-Veress, Adv. Colloid Interface Sci. 94 (2001) $167-196$. 